\section{WEDGE BRONCHOPLASTIC LOBECTOMY}

To the Editor:

"Wedge bronchoplastic lobectomy for non-small cell lung cancer as an alternative to sleeve lobectomy" is an interesting report that raises various issues. ${ }^{1}$ The authors presented an institutional retrospective study of a respectable number of wedge bronchoplastic lobectomies. They concluded that this procedure is feasible, safe, oncologically acceptable, and, finally, an appropriate alternative to sleeve lobectomy. In our understanding, the main issue is the preservation of a bronchial segment, which should ensure the vascular supply to the distal stump. This technical solution should prevent bronchopleural fistula and circumferential stricture, 2 frightening complications after sleeve lobectomy.

The topics of interest are whether the vascular supply will be useful to anastomotic healing; whether the geometry is relevant in the bronchial suture; and whether the local oncologic situation is important.

It is well known that an appropriate vascular supply is essential to a visceral anastomosis; however, the bronchus could be considered a peculiar case for at least 2 reasons. First, the bronchial wall is mainly composed of cartilage ( 1 of the less vascularized tissues in the human body); and second, bronchial function is essentially related to the very bronchial shape, rather than any energy-consuming activity. The major concern about the vascular supply in the bronchial anastomosis comes from our experience in lung transplantation and repeat transplantation. $^{2}$ During lung transplantation, any attempt to reconstruct the bronchial vascular supply was abandoned decades ago. In addition, the donor bronchial stump will have been ischemic for hours before surgery, the recipient stump diameters are frequently incongruent with the donor diameter, the patients will be hypoxic by definition, and corticosteroids are generously administered. Despite these negative factors, the rates of bronchopleural fistula in lung transplantation and sleeve lobectomy have been similar. Considering these brief reflections, the preservation of a segment of the bronchus, with the intent of improving the vascular supply to the anastomosis, seems redundant. In contrast, such a bronchial segment could constitute an obstacle to approximate the 2 stumps. The authors suggested performing an outside folding of the redundant bronchial tissue. We believe that a sharp perpendicular cut will allow for an appropriate approximation of the stumps and a geometric reconstruction similar to the physiologic one. These expedients, in association with a tension-free suture, should be the basis for a good anastomosis, even during thoracoscopic procedures. $^{3}$ Because the results from frozen section analysis could be unreliable, the local oncologic situation is probably the major concern with sleeve lobectomy. This is the reason we prefer a circumferential cut at a good distance from the tumor.

Wedge bronchoplastic lobectomy is surely a valuable technical procedure for a small minority of cases; than, cui prodest the preservation of a redundant fragment of bronchial wall? Is it a psychological help?

Mario Nosotti, $M D$

Matilde De Simone, $M D, P h D$

Ugo Cioffi, $M D, P h D$

Department of Surgery

Fondazione IRCCS Ca' Granda

Ospedale Maggiore Policlinico

University of Milan

Milan, Italy

\section{References}

1. Park SY, Lee HS, Jang HJ, Joo J, Kim MS, Lee JM, et al. Wedge bronchoplastic lobectomy for non-small cell lung cancer as an alternative to sleeve lobectomy. J Thorac Cardiovasc Surg. 2012;143:825-31.e3.

2. Nosotti M, Rosso L, Tosi D, Palleschi A, Mendogni P, Crotti S, et al. Late lung retransplantation using extracorporeal membrane oxygenation as a bridge: case report. Transplant Proc. 2011; 43:1198-200.
3. Santambrogio L, Cioffi U, De Simone M, Rosso L, Ferrero S, Giunta A. Video-assisted sleeve lobectomy for mucoepidermoid carcinoma of the left lower lobar bronchus: a case report. Chest. 2002;121:635-6.

http://dx.doi.org/10.1016/

j.jtcvs.2012.04.016

\section{Reply to the Editor:}

We appreciate the letter by Nosotti and colleagues regarding our report, and we are delighted with their thoughtful insights into our results. ${ }^{1}$ Despite their assertions that, based on their experience with lung transplantation, the redundant segment of the bronchus might not be helpful for anastomotic healing during wedge bronchoplastic lobectomy, we have different opinions.

Dr Grillo stated that the 2 feared complications after sleeve lobectomy-bronchostenosis and bronchovascular fistula-showed a vanishing incidence when the procedure is performed in accordance with the principles of precise dissection, blood supply preservation, meticulous anastomosis, elimination of anastomotic tension, anastomotic buttressing, and the interposition of viable tissue between bronchial and vascular structures. $^{2}$ During lung transplantation, the maintenance of the airway blood supply is primarily related to the preservation of all available bronchopulmonary collateral circulation and maximizing reversed flow through this circuit. Keeping "excess" peribronchial tissue intact, including any attached nodal and pericardial tissues, is valuable in this respect. However, during lung cancer surgery, complete mediastinal lymph node dissection and aggressive manipulation of the peribronchial tissues are essential to maintain the oncologic principles. Furthermore, patients requiring bronchoplastic lobectomy are generally older men and heavy smokers with squamous cell histologic features. Our study $^{1}$ showed that the patient population was primarily characterized by male sex $(92 \%)$, stage $\mathrm{N} 1$ or $\mathrm{N} 2$ 
disease $(65 \%)$, and squamous cell carcinoma $(80 \%)$. Although all thoracic surgeons maintain these principles during sleeve lobectomy for lung cancer, bronchostenosis and fistula remain fearful complications.

The etiology of airway complications in most cases is related to airway ischemia. The resultant ischemia of the airway can lead to mucosal ulceration and subsequent granulation tissue formation or transmural necrosis. Preservation of the airway blood supply is the most critical factor for obtaining a successful airway anastomosis in bronchoplasty patients. The primary blood supply to the common sites of anastomosis, including the distal trachea and main bronchus, is derived from the bronchial circulation. The bronchial arteries form the peribronchial arterial bronchial plexus. These plexuses also give rise to small arterioles that penetrate the muscular layer to reach the bronchial mucosa, where they form the submucosal plexus. ${ }^{3}$ However, after sleeve lobectomy, the systemic connection to the bronchial arteries is usually not restored. A flow reversal from the pulmonary circulation into the bronchial circulation by way of the bronchial venous network occurs, resulting in perfusion of the peribronchial and submucosal plexuses of the airway. ${ }^{4}$ Compromising the bronchopulmonary collateral flow can lead to ischemic sequelae at the site of anastomosis. Clinical experience suggests that the amount of perfusion achieved by this flow reversal from the pulmonary circulation to the bronchial circulation is sufficient to maintain airway viability and allow for anastomotic healing. During bronchial dissection, we can examine the blood supply of the resected bronchus. If the bronchial segment is saved by bronchial wedge resection, the blood supply can also be maintained by way of the submucosal plexus in the remnant bronchus.

Regarding the geometric issue, we must see both the forest and the trees. Lobectomy leads to the geometric alteration of the bronchus by the expansion of the remnant lung with resolution of the space. In particular, for upper lobectomies, in which sleeve lobectomy is commonly performed, the main bronchus and main pulmonary artery are frequently angulated to resolve the space problem. In this study, approximately $70 \%$ of the patients who underwent upper lobectomy. Sleeve lobectomy appears to be a geometric reconstruction during deflation of the lungs in the operative field. However, after chest closure with lung inflation, the intraoperative geometric stability can deteriorate, with increased tension at the counterpart site of the resected bronchus. However, a transverse or oblique approximation of the bronchus and retaining the counterpart bronchus during wedge bronchoplasty can be more tolerable to this geometric alteration. An intraoperative bronchoscopic confirmation of the patency at the anastomotic site is thought to be enough to perform a successful anastomosis.

Oncologically, we have already shown acceptable oncologic results after wedge bronchoplastic lobectomy. Dr Nosotti's case was a 15-year-old girl with low-grade mucoepidermoid carcinoma of the left lower lobar bronchus who underwent a video-assisted thoracic surgical sleeve lobectomy. ${ }^{5}$ However, I believe this type of polypoid tumor protruding into the main bronchus without mucosal spread is the best indication for wedge bronchoplastic lobectomy, because it is the easier and simpler method and does not have the potential for oncologic compromise. The usefulness of wedge bronchoplastic lobectomy for lung cancer is based on anatomic, geometric, and oncologic evidence, as well as a surgeon's psychological health.

Hyun-Sung Lee, MD, PhD Seong Yong Park, MD Center for Lung Cancer Research Institute and Hospital,
National Cancer Center

Goyang, Gyeonggi, Republic of Korea

\section{References}

1. Park SY, Lee HS, Jang HJ, Joo J, Kim MS, Lee JM, et al. Wedge bronchoplastic lobectomy for nonsmall cell lung cancer as an alternative to sleeve lobectomy. J Thorac Cardiovasc Surg. 2012;143: 825-31.

2. Mathisen DJ. Main and lobar bronchoplasty. In: Grillo HC, ed. Surgery of the trachea and bronchi. Hamilton, ON: BC Decker; 2004. p. 619-29.

3. Salassa JR, Pearson BW, Payne WS. Gross and microscopic blood supply of the trachea. Ann Thorac Surg. 1977;24:100-7.

4. Ladowski JS, Hardesty RL, Griffith BP. The pulmonary artery blood supply to the supracarinal trachea. J Heart Transplant. 1984;4:40-2.

5. Santambrogio L, Cioffi U, De Simone M, Rosso L, Ferrero S, Giunta A. Video-assisted sleeve lobectomy for mucoepidermoid carcinoma of the left lower lobar bronchus: a case report. Chest. 2002; 121:635-6.

http://dx.doi.org/10.1016/ j.jtcvs.2012.05.004

\section{CAN THE SUCCESS OF TRANSCATHETER AORTIC VALVE IMPLANTATION BE INCREASED?}

\section{To the Editor:}

We congratulate the authors on their study. ${ }^{1}$ After application of a size 26 Sapien valve to one third of the patients, the postoperative effective orifice area was noted to be $1.67 \mathrm{~cm}^{2}$ and the geometric size was $2.5 \mathrm{~cm}^{2}$ in the size 21 Sapien valve. This suggests that the balloon should be inflated more. Instead of "hinge-tohinge" or "virtual ring" calculations, we suggest that an experienced cardiac surgeon can decide which leaflet should be dilated to what degree, while inflating the valve using computed tomography or echocardiography, because the Sapien valve is located at the leaflet and not at the annulus.

Unbehaun and colleagues ${ }^{2}$ examined transvalvular insufficiency in this study. If the location of the prosthesis over the leaflet is asymmetric, a transvalvular gradient can occur. Transvalvular regurgitation necessitates balloon dilation.

The shapes of the Edwards valves is different from that of the CoreValve. 